\title{
Dilation and Erosion of Spatial Bipolar Fuzzy Sets
}

\author{
Isabelle Bloch \\ GET - Télécom Paris (ENST) \\ Dept. TSI - CNRS UMR 5141 LTCI \\ 46 rue Barrault, 75013 Paris, France \\ Isabelle.Bloch@enst.fr
}

\begin{abstract}
Bipolarity has not been much exploited in the spatial domain yet, although it has many features to manage imprecise and incomplete information that could be interesting in this domain. This paper is a first step to address this issue, and we propose to define mathematical morphology operations on bipolar fuzzy sets (or equivalently interval valued fuzzy sets or intuitionistic fuzzy sets).
\end{abstract}

\section{Introduction}

In many domains, it is important to be able to deal with bipolar information 11. Positive information represents what is granted to be possible (for instance because it has already been observed or experienced), while negative information represents what is impossible (or forbidden, or surely false). This view is supported by studies in cognitive psychology (e.g. [2]), which show that two independent types of information (positive and negative) are processed separately in the brain. The intersection of the positive information and the negative information has to be empty in order to achieve consistency of the representation, and their union does not necessarily covers the whole underlying space (i.e. there is no direct duality between both types of information).

This domain has recently motivated work in several directions. In particular, fuzzy and possibilistic formalisms for bipolar information have been proposed [1. Interestingly enough, they are directly linked to intuitionistic fuzzy sets [3], interval-valued fuzzy sets [4] and vague sets, as shown e.g. in [56].

When dealing with spatial information, in image processing or for spatial reasoning applications, this bipolarity also occurs. For instance, when assessing the position of an object in space, we may have positive information expressed as a set of possible places, and negative information expressed as a set of impossible places (for instance because they are occupied by other objects). As another example, let us consider spatial relations. Human beings consider "left" and "right" as opposite relations. But this does not mean that one of them is the negation of the other one. The semantics of "opposite" captures a notion of symmetry rather than a strict complementation. In particular, there may be positions which are considered neither to the right nor to the left of some reference

F. Masulli, S. Mitra, and G. Pasi (Eds.): WILF 2007, LNAI 4578, pp. $385+3932007$.

(C) Springer-Verlag Berlin Heidelberg 2007 
object, thus leaving room for some indetermination [7]. This corresponds to the idea that the union of positive and negative information does not cover all the space.

To our knowledge, bipolarity has not been much exploited in the spatial domain. The above considerations are the motivation for the present work, which aims at filling this gap by proposing formal models to manage spatial bipolar information. Additionally, imprecision has to be included, since it is an important feature of spatial information, related either to the objects themselves or to the spatial relations between them. More specifically, we consider bipolar fuzzy sets, and propose definitions of mathematical morphology operators (dilation and erosion) on these representations. To our knowledge, this is a completely new contribution in the domain of bipolar fuzzy sets.

In Section 2, we recall some definitions on bipolar fuzzy sets. Then we introduce definitions of algebraic dilations and erosions of bipolar fuzzy sets in Section 3. In the spatial domain, specific forms of these operators, involving a structuring element, are particularly interesting 8]. They are called morphological dilation and erosion. Morphological erosion is then defined in Section 4. Two forms of morphological dilations are proposed in Section 5, either based on duality or on adjunction. Properties are given in Section 6 .

\section{Preliminaries}

Let $\mathcal{S}$ be the underlying space (the spatial domain for spatial information processing). A bipolar fuzzy set on $\mathcal{S}$ is defined by a pair of functions $(\mu, \nu)$ such that $\forall x \in \mathcal{S}, \mu(x)+\nu(x) \leq 1$. Note that a bipolar fuzzy set is equivalent to an intuitionistic fuzzy set 3 , as shown in [5. It is also equivalent to an intervalvalued fuzzy set [4], where the interval at each point $x$ is $[\mu(x), 1-\nu(x)][5]$. Although there has been a lot of discussion about terminology in this domain recently [59], we use the bipolarity terminology in this paper, for its appropriate semantics, as explained in our motivation. For each point $x, \mu(x)$ defines the degree to which $x$ belongs to the bipolar fuzzy set (positive information) and $\nu(x)$ the non-membership degree (negative information). This formalism allows representing both bipolarity and fuzziness.

Let us consider the set of pairs of numbers $(a, b)$ in $[0,1]$ such that $a+b \leq 1$. This set is a complete lattice, for the partial order defined as [10]:

$$
\left(a_{1}, b_{1}\right) \preceq\left(a_{2}, b_{2}\right) \text { iff } a_{1} \leq a_{2} \text { and } b_{1} \geq b_{2} .
$$

The greatest element is $(1,0)$ and the smallest element is $(0,1)$. The supremum and infimum are respectively defined as:

$$
\begin{aligned}
& \left(a_{1}, b_{1}\right) \vee\left(a_{2}, b_{2}\right)=\left(\max \left(a_{1}, a_{2}\right), \min \left(b_{1}, b_{2}\right)\right), \\
& \left(a_{1}, b_{1}\right) \wedge\left(a_{2}, b_{2}\right)=\left(\min \left(a_{1}, a_{2}\right), \max \left(b_{1}, b_{2}\right)\right) .
\end{aligned}
$$


The partial order $\preceq$ induces a partial order on the set of bipolar fuzzy sets:

$$
\left(\mu_{1}, \nu_{1}\right) \preceq\left(\mu_{2}, \nu_{2}\right) \text { iff } \forall x \in \mathcal{S}, \mu_{1}(x) \leq \mu_{2}(x) \text { and } \nu_{1}(x) \geq \nu_{2}(x) .
$$

Note that this corresponds to the inclusion on intuitionistic fuzzy sets defined in 3. Similarly the supremum and the infimum are equivalent to the intuitionistic union and intersection.

It follows that, if $\mathcal{B}$ denotes the set of bipolar fuzzy sets on $\mathcal{S},(\mathcal{B}, \preceq)$ is a complete lattice.

\section{Algebraic Dilation and Erosion of Bipolar Fuzzy Sets}

Once we have a complete lattice, it is easy to define algebraic dilations and erosions on this lattice.

Definition 1. A dilation is an operator $\delta$ from $\mathcal{B}$ into $\mathcal{B}$ that commutes with the supremum:

$$
\delta\left((\mu, \nu) \vee\left(\mu^{\prime}, \nu^{\prime}\right)\right)=\delta((\mu, \nu)) \vee \delta\left(\left(\mu^{\prime}, \nu^{\prime}\right)\right)
$$

An erosion is an operator $\varepsilon$ from $\mathcal{B}$ into $\mathcal{B}$ that commutes with the infimum:

$$
\varepsilon\left((\mu, \nu) \wedge\left(\mu^{\prime}, \nu^{\prime}\right)\right)=\varepsilon((\mu, \nu)) \wedge \varepsilon\left(\left(\mu^{\prime}, \nu^{\prime}\right)\right) .
$$

The following result is useful for proving the next results.

\section{Lemma 1}

$$
(\mu, \nu) \preceq\left(\mu^{\prime}, \nu^{\prime}\right) \Leftrightarrow\left\{\begin{array}{l}
(\mu, \nu) \vee\left(\mu^{\prime}, \nu^{\prime}\right)=\left(\mu^{\prime}, \nu^{\prime}\right) \\
(\mu, \nu) \wedge\left(\mu^{\prime}, \nu^{\prime}\right)=(\mu, \nu)
\end{array}\right.
$$

The following results are directly derived from the properties of complete lattices [1].

Proposition 1. The following results hold:

$-\delta$ and $\varepsilon$ are increasing operators;

$-\delta((0,1))=(0,1)$;

$-\varepsilon((1,0))=(1,0)$;

- by denoting $\left(\mu_{x}, \nu_{x}\right)$ the canonical bipolar fuzzy set associated with $(\mu, \nu)$ and $x$ such that $\left(\mu_{x}, \nu_{x}\right)(x)=(\mu(x), \nu(x))$ and $\forall y \in \mathcal{S} \backslash\{x\},\left(\mu_{x}, \nu_{x}\right)(y)=(0,1)$, we have $(\mu, \nu)=\bigvee_{x}\left(\mu_{x}, \nu_{x}\right)$ and $\delta((\mu, \nu))=\bigvee_{x} \delta\left(\left(\mu_{x}, \nu_{x}\right)\right)$

The last result leads to morphological operators in case $\delta\left(\left(\mu_{x}, \nu_{x}\right)\right)$ has the same "shape" everywhere (and is then a bipolar fuzzy structuring element).

Definition 2. A pair of operators $(\varepsilon, \delta)$ defines an adjunction on $(\mathcal{B}, \preceq)$ iff:

$$
\forall(\mu, \nu) \in \mathcal{B}, \forall\left(\mu^{\prime}, \nu^{\prime}\right) \in \mathcal{B}, \delta((\mu, \nu)) \preceq\left(\mu^{\prime}, \nu^{\prime}\right) \Leftrightarrow(\mu, \nu) \preceq \varepsilon\left(\left(\mu^{\prime}, \nu^{\prime}\right)\right)
$$


Again we can derive a series of results from the properties of complete lattices and adjunctions.

Proposition 2. If a pair of operators $(\varepsilon, \delta)$ defines an adjunction, then the following results hold:

$-\delta$ is a dilation and $\varepsilon$ is an erosion, in the sense of Definition 1;

- $\delta \varepsilon \preceq I d$, where Id denotes the identity mapping on $\mathcal{B}$;

$-I d \preceq \varepsilon \delta$;

$-\delta \varepsilon \delta \varepsilon=\delta \varepsilon$ and $\varepsilon \delta \varepsilon \delta=\varepsilon \delta$, i.e. the composition of a dilation and an erosion are idempotent operators.

The following representation result also holds.

Proposition 3. If $\varepsilon$ is an increasing operator, it is an algebraic erosion if and only if there exists $\delta$ such that $(\varepsilon, \delta)$ is an adjunction. The operator $\delta$ is then an algebraic dilation and can be expressed as:

$$
\delta((\mu, \nu))=\inf \left\{\left(\mu^{\prime}, \nu^{\prime}\right) \in \mathcal{B},(\mu, \nu) \preceq \varepsilon\left(\left(\mu^{\prime}, \nu^{\prime}\right)\right)\right\} .
$$

A similar representation result holds for erosion.

\section{Morphological Erosion of Bipolar Fuzzy Sets}

We now assume that $\mathcal{S}$ is an affine space (or at least a space on which translations can be defined). The general principle underlying morphological erosions is to translate the structuring element at every position in space and check if this translated structuring element is included in the original set 8 . This principle has also been used in the main extensions of mathematical morphology to fuzzy sets 1213 14 1516]. Similarly, defining morphological erosions of bipolar fuzzy sets, using bipolar fuzzy structuring elements, requires to define a degree of inclusion between bipolar fuzzy sets. Such inclusion degrees have been proposed in the context of intuitionistic fuzzy sets in [17. With our notations, a degree of inclusion of a bipolar fuzzy set $\left(\mu^{\prime}, \nu^{\prime}\right)$ in another bipolar fuzzy set $(\mu, \nu)$ is defined as:

$$
\inf _{x \in \mathcal{S}} I\left(\left(\mu^{\prime}(x), \nu^{\prime}(x)\right),(\mu(x), \nu(x))\right)
$$

where $I$ is an implication operator. Two types of implication are used in 1718 , one derived from an intuitionistic (or bipolar) t-conorm $\perp$, and one derived from a residuation principle from an intuitionistic t-norm $T$ :

$$
\begin{gathered}
I_{N}\left(\left(a_{1}, b_{1}\right),\left(a_{2}, b_{2}\right)\right)=\perp\left(\left(b_{1}, a_{1}\right),\left(a_{2}, b_{2}\right)\right), \\
I_{R}\left(\left(a_{1}, b_{1}\right),\left(a_{2}, b_{2}\right)\right)=\sup \left\{\left(a_{3}, b_{3}\right), \top\left(\left(a_{1}, b_{1}\right),\left(a_{3}, b_{3}\right)\right) \preceq\left(a_{2}, b_{2}\right)\right\}
\end{gathered}
$$

where $\left(a_{i}, b_{i}\right)$ are numbers in $[0,1]$ such that $a_{i}+b_{i} \leq 1$ and $\left(b_{i}, a_{i}\right)$ is the standard negation of $\left(a_{i}, b_{i}\right)$.

Two types of t-norms and t-conorms are considered in [17 and will be considered here as well: 
1. operators called t-representable t-norms and t-conorms, which can be expressed using usual t-norms $t$ and t-conorms $T$ :

$$
\begin{aligned}
& \top\left(\left(a_{1}, b_{1}\right),\left(a_{2}, b_{2}\right)\right)=\left(t\left(a_{1}, a_{2}\right), T\left(b_{1}, b_{2}\right)\right), \\
& \perp\left(\left(a_{1}, b_{1}\right),\left(a_{2}, b_{2}\right)\right)=\left(T\left(a_{1}, a_{2}\right), t\left(b_{1}, b_{2}\right)\right) .
\end{aligned}
$$

2. Lukasiewicz operators, which are not t-representable:

$$
\begin{aligned}
& \top_{W}\left(\left(a_{1}, b_{1}\right),\left(a_{2}, b_{2}\right)\right)=\left(\max \left(0, a_{1}+a_{2}-1\right), \min \left(1, b_{1}+1-a_{2}, b_{2}+1-a_{1}\right)\right), \\
& \perp_{W}\left(\left(a_{1}, b_{1}\right),\left(a_{2}, b_{2}\right)\right)=\left(\min \left(1, a_{1}+1-b_{2}, a_{2}+1-b_{1}\right), \max \left(0, b_{1}+b_{2}-1\right)\right) .
\end{aligned}
$$

The two types of implication coincide for the Lukasiewicz operators, as shown in [10.

Based on these concepts, we can now propose a definition for morphological erosion.

Definition 3. Let $\left(\mu_{B}, \nu_{B}\right)$ be a bipolar fuzzy structuring element (in $\left.\mathcal{B}\right)$. The erosion of any $(\mu, \nu)$ in $\mathcal{B}$ by $\left(\mu_{B}, \nu_{B}\right)$ is defined from an implication $I$ as:

$$
\forall x \in \mathcal{S}, \varepsilon_{\left(\mu_{B}, \nu_{B}\right)}((\mu, \nu))(x)=\inf _{y \in \mathcal{S}} I\left(\left(\mu_{B}(y-x), \nu_{B}(y-x)\right),(\mu(y), \nu(y))\right) .
$$

\section{Morphological Dilation of Bipolar Fuzzy Sets}

Dilation can be defined based on a duality principle or based on the adjunction property. Both approaches have been developed in the case of fuzzy sets, and the links between them and the conditions for their equivalence have been proved in [19. Similarly we consider both approaches to define morphological dilation on $\mathcal{B}$.

Dilation by duality. The duality principle states that the dilation is equal to the complementation of the erosion, by the same structuring element, applied to the complementation of the original set. Applying this principle to the bipolar fuzzy sets using a complementation $c$ (typically the standard negation $c((a, b))=(b, a)$ ) leads to the following definition of morphological bipolar dilation.

Definition 4. Let $\left(\mu_{B}, \nu_{B}\right)$ be a bipolar fuzzy structuring element. The dilation of any $(\mu, \nu)$ in $\mathcal{B}$ by $\left(\mu_{B}, \nu_{B}\right)$ is defined from erosion by duality as:

$$
\delta_{\left(\mu_{B}, \nu_{B}\right)}((\mu, \nu))=c\left[\varepsilon_{\left(\mu_{B}, \nu_{B}\right)}(c((\mu, \nu)))\right] .
$$

Dilation by adjunction. Let us now consider the adjunction principle, as in the general algebraic case. An adjunction property can also be expressed between a bipolar t-norm and the corresponding residual implication as follows:

$$
\top\left(\left(a_{1}, b_{1}\right),\left(a_{3}, b_{3}\right)\right) \preceq\left(a_{2}, b_{2}\right) \Leftrightarrow\left(a_{3}, b_{3}\right) \preceq I\left(\left(a_{1}, b_{1}\right),\left(a_{2}, b_{2}\right)\right)
$$

with $I\left(\left(a_{1}, b_{1}\right),\left(a_{2}, b_{2}\right)\right)=\sup \left\{(\alpha, \beta), \alpha+\beta \leq 1, \top\left(\left(a_{1}, b_{1}\right),(\alpha, \beta)\right) \preceq\left(a_{2}, b_{2}\right)\right\}$. 
Definition 5. Using a residual implication for the erosion for a bipolar t-norm $\top$, the bipolar fuzzy dilation, adjoint of the erosion, is defined as:

$$
\begin{aligned}
& \delta_{\left(\mu_{B}, \nu_{B}\right)}((\mu, \nu))(x)=\inf \{\left.\left(\mu^{\prime}, \nu^{\prime}\right)(x),(\mu, \nu)(x) \preceq \varepsilon_{\left(\mu_{B}, \nu_{B}\right)}\left(\left(\mu^{\prime}, \nu^{\prime}\right)\right)(x)\right\} \\
&=\sup _{y \in \mathcal{S}} \top\left(\left(\mu_{B}(x-y), \nu_{B}(x-y)\right),(\mu(y), \nu(y))\right) .
\end{aligned}
$$

Links between both approaches. It is easy to show that the bipolar Lukasiewicz operators are adjoint, according to Equation 19. It has been shown in 17] that the adjoint operators are all derived from the Lukasiewicz operator, using a continuous bijective permutation on $[0,1]$. Hence equivalence between both approaches can be achieved only for this class of operators.

\section{Properties and Interpretation}

Proposition 4. All definitions are consistent: they actually provide bipolar fuzzy sets of $\mathcal{B}$.

Let us first consider the implication defined from a t-representable bipolar tconorm. Then the erosion writes:

$$
\begin{aligned}
\varepsilon_{\left(\mu_{B}, \nu_{B}\right)}((\mu, \nu))(x) & =\inf _{y \in \mathcal{S}} \perp\left(\left(\nu_{B}(y-x), \mu_{B}(y-x)\right),(\mu(y), \nu(y))\right) \\
& =\inf _{y \in \mathcal{S}}\left(T\left(\left(\nu_{B}(y-x), \mu(y)\right), t\left(\mu_{B}(y-x), \nu(y)\right)\right)\right. \\
& =\inf _{y \in \mathcal{S}} T\left(\left(\nu_{B}(y-x), \mu(y)\right), \sup _{y \in \mathcal{S}} t\left(\mu_{B}(y-x), \nu(y)\right)\right) .
\end{aligned}
$$

This resulting bipolar fuzzy set has a membership function which is exactly the fuzzy erosion of $\mu$ by the fuzzy structuring element $1-\nu_{B}$, according to the definitions of [12. The non-membership function is exactly the dilation of the fuzzy set $\nu$ by the fuzzy structuring element $\mu_{B}$.

Let us now consider the derived dilation, based on the duality principle. Using the standard negation, it writes:

$$
\delta_{\left(\mu_{B}, \nu_{B}\right)}((\mu, \nu))(x)=\left(\sup _{y \in \mathcal{S}} t\left(\mu_{B}(x-y), \mu(y)\right), \inf _{y \in \mathcal{S}} T\left(\left(\nu_{B}(x-y), \nu(y)\right)\right) .\right.
$$

The first term (membership function) is exactly the fuzzy dilation of $\mu$ by $\mu_{B}$, while the second one (non-membership function) is the fuzzy erosion of $\nu$ by $1-\nu_{B}$, according to the definitions of 12 .

This observation has a nice interpretation. Let $(\mu, \nu)$ represent a spatial bipolar fuzzy set, where $\mu$ is a positive information for the location of an object for instance, and $\nu$ a negative information for this location. A bipolar structuring element can represent additional imprecision on the location, or additional possible locations. Dilating $(\mu, \nu)$ by this bipolar structuring element amounts to dilate $\mu$ by $\mu_{B}$, i.e. the positive region is extended by an amount represented by the positive information encoded in the structuring element. On the contrary, 
the negative information is eroded by the complement of the negative information encoded in the structuring element. This corresponds well to what would be intuitively expected in such situations. A similar interpretation can be provided for the bipolar fuzzy erosion.

From these expressions it is easy to prove the following result.

Proposition 5. In case the bipolar fuzzy sets are usual fuzzy sets (i.e. $\nu=1-\mu$ and $\left.\nu_{B}=1-\mu_{B}\right)$, the definitions lead to the usual definitions of fuzzy dilations and erosions. Hence they are also compatible with classical morphology in case $\mu$ and $\mu_{B}$ are crisp.

Let us now consider the implication derived from the Lukasiewicz bipolar operators (Equations [15] and 16). The erosion and dilation write:

$$
\begin{aligned}
& \forall x \in \mathcal{S}, \varepsilon_{\left(\mu_{B}, \nu_{B}\right)}((\mu, \nu))(x)= \\
& \inf _{y \in \mathcal{S}}\left(\min \left(1, \mu(y)+1-\mu_{B}(y-x), \nu_{B}(y-x)+1-\nu(y)\right), \max \left(0, \nu(y)+\mu_{B}(y-x)-1\right)\right)= \\
& \left(\inf _{y \in \mathcal{S}} \min \left(1, \mu(y)+1-\mu_{B}(y-x), \nu_{B}(y-x)+1-\nu(y)\right), \sup _{y \in \mathcal{S}} \max \left(0, \nu(y)+\mu_{B}(y-x)-1\right)\right), \\
& \forall x \in \mathcal{S}, \delta_{\left(\mu_{B}, \nu_{B}\right)}((\mu, \nu))(x)= \\
& \left(\sup _{y \in \mathcal{S}} \max \left(0, \mu(y)+\mu_{B}(x-y)-1\right), \inf _{y \in \mathcal{S}} \min \left(1, \nu(y)+1-\mu_{B}(x-y), \nu_{B}(x-y)+1-\mu(y)\right) .\right.
\end{aligned}
$$

Proposition 6. If the bipolar fuzzy sets are usual fuzzy sets (i.e. $\nu=1-\mu$ and $\left.\nu_{B}=1-\mu_{B}\right)$, the definitions based on the Lukasiewicz operators are equivalent to the fuzzy erosion defined as in [12] by the infimum of a t-conorm for the classical Lukasiewicz t-conorm, and to the fuzzy dilation defined by the supremum of a t-norm for the classical Lukasiewicz t-norm, respectively.

Proposition 7. The proposed definitions of bipolar fuzzy dilations and erosions commute respectively with the supremum and the infinum of the lattice $(\mathcal{B}, \preceq)$.

Proposition 8. The bipolar fuzzy dilation is extensive (i.e. $(\mu, \nu) \preceq$ $\left.\delta_{\left(\mu_{B}, \nu_{B}\right)}((\mu, \nu))\right)$ and the bipolar fuzzy erosion is anti-extensive (i.e. $\left.\varepsilon_{\left(\mu_{B}, \nu_{B}\right)}((\mu, \nu)) \preceq(\mu, \nu)\right)$ if and only if $\left(\mu_{B}, \nu_{B}\right)(0)=(1,0)$, where 0 is the origin of the space $\mathcal{S}$ (i.e. the origin completely belongs to the structuring element, without any indetermination).

Note that this condition is equivalent to the conditions on the structuring element found in classical and fuzzy morphology to have extensive dilations and anti-extensive erosions 812.

Proposition 9. If the dilation if defined from a t-representable $t$-norm, the following iterativity property holds:

$$
\delta_{\left(\mu_{B}, \nu_{B}\right)}\left(\delta_{\left(\mu_{B}^{\prime}, \nu_{B}^{\prime}\right)}((\mu, \nu))\right)=\delta_{\left(\delta_{\mu_{B}}\left(\mu_{B}^{\prime}\right), 1-\delta_{\left(1-\nu_{B}\right)}\left(1-\nu_{B}^{\prime}\right)\right)}((\mu, \nu)) .
$$




\section{Conclusion}

New concepts on bipolar fuzzy sets are introduced in this paper, in particular algebraic and morphological dilations and erosions, for which good properties are proved and nice interpretations in terms of bipolarity in spatial reasoning can be derived. Further work aims at exploiting these new operations in concrete problems of spatial reasoning, in particular for handling the bipolarity nature of some spatial relations.

\section{References}

1. Dubois, D., Kaci, S., Prade, H.: Bipolarity in Reasoning and Decision, an Introduction. In: International Conference on Information Processing and Management of Uncertainty, IPMU'04, Perugia, Italy, pp. 959-966 (2004)

2. Cacioppo, J.T., Gardner, W.L., Berntson, G.G.: Beyond Bipolar Conceptualization and Measures: The Case of Attitudes and Evaluative Space. Personality and Social Psychology Review 1, 3-25 (1997)

3. Atanassov, K.T.: Intuitionistic Fuzzy Sets. Fuzzy Sets and Systems 20, 87-96 (1986)

4. Zadeh, L.A.: The Concept of a Linguistic Variable and its Application to Approximate Reasoning. Information Sciences 8, 199-249 (1975)

5. Dubois, D., Gottwald, S., Hajek, P., Kacprzyk, J., Prade, H.: Terminology Difficulties in Fuzzy Set Theory - The Case of "Intuitionistic Fuzzy Sets". Fuzzy Sets and Systems 156, 485-491 (2005)

6. Bustince, H., Burillo, P.: Vague Sets are Intuitionistic Fuzzy Sets. Fuzzy Sets and Systems 79, 403-405 (1996)

7. Bloch, I.: Fuzzy Relative Position between Objects in Image Processing: a Morphological Approach. IEEE Transactions on Pattern Analysis and Machine Intelligence 21, 657-664 (1999)

8. Serra, J.: Image Analysis and Mathematical Morphology. Academic Press, London (1982)

9. Atanassov, K.T., Dubois, D., Gottwald, S., Hajek, P., Kacprzyk, J., Prade's papers, H.: Terminology Difficulties in Fuzzy Set Theory - The Case of "Intuitionistic Fuzzy Sets". Fuzzy Sets and Systems 156, 496-499 (2005)

10. Cornelis, C., Kerre, E.: Inclusion Measures in Intuitionistic Fuzzy Sets. In: Nielsen, T.D., Zhang, N.L. (eds.) ECSQARU 2003. LNCS (LNAI), vol. 2711, pp. 345-356. Springer, Heidelberg (2003)

11. Heijmans, H.J.A.M., Ronse, C.: The Algebraic Basis of Mathematical Morphology - Part I: Dilations and Erosions. Computer Vision, Graphics and Image Processing 50, 245-295 (1990)

12. Bloch, I., Maître, H.: Fuzzy Mathematical Morphologies: A Comparative Study. Pattern Recognition 28, 1341-1387 (1995)

13. Sinha, D., Dougherty, E.R.: Fuzzification of Set Inclusion: Theory and Applications. Fuzzy Sets and Systems 55, 15-42 (1993)

14. de Baets, B.: Fuzzy Morphology: a Logical Approach. In: Ayyub, B., Gupta, M. (eds.) Uncertainty in Engineering and Sciences: Fuzzy Logic, Statistics and Neural Network Approach, pp. 53-67. Kluwer Academic, Boston, MA (1997) 
15. Nachtegael, M., Kerre, E.E.: Classical and Fuzzy Approaches towards Mathematical Morphology. In: Kerre, E.E., Nachtegael, M. (eds.) Fuzzy Techniques in Image Processing. Studies in Fuzziness and Soft Computing, pp. 3-57. Physica-Verlag, Springer, Heidelberg (2000)

16. Deng, T.Q., Heijmans, H.: Grey-Scale Morphology Based on Fuzzy Logic. Journal of Mathematical Imaging and Vision 16, 155-171 (2002)

17. Deschrijver, G., Cornelis, C., Kerre, E.: On the Representation of Intuitionistic Fuzzy t-Norms and t-Conorms. IEEE Transactions on Fuzzy Systems 12, 45-61 (2004)

18. Cornelis, C., Deschrijver, G., Kerre, E.: Implication in Intuitionistic Fuzzy and Interval-Valued Fuzzy Set Theory: Construction, Classification, Application. International Journal of Approximate Reasoning 35, 55-95 (2004)

19. Bloch, I.: Duality vs Adjunction and General Form for Fuzzy Mathematical Morphology. In: Bloch, I., Petrosino, A., Tettamanzi, A.G.B. (eds.) WILF 2005. LNCS (LNAI), vol. 3849, pp. 354-361. Springer, Heidelberg (2006) 